\title{
Towards Geometric Control of Max-Plus Linear Systems with Applications to Manufacturing Systems
}

\author{
Laurent Hardouin, Mehdi Lhommeau, Ying Shang
}

\begin{abstract}
The max-plus linear systems have been studied for almost three decades, however, a well-established system theory on such specific systems is still an on-going research. The geometric control theory in particular was proposed as the future direction for max-plus linear systems by Cohen et al. This paper reports upon recent investigations on the disturbance decoupling problem for max-plus linear systems, which is the standard geometric control problem originated by W. M. Wonham. Different concepts of the disturbance decoupling problem are introduced, as well as the corresponding solvability conditions and controller synthesis procedures. The main results can be used in manufacturing systems, queueing networks, and power system networks for fault detection and system breakdown prevention.
\end{abstract}

\section{INTRODUCTION}

Max-plus linear systems have been used in communication networks [13], genetic regulatory networks [8], [10], and queueing systems [1]. The fundamental problems for maxplus linear systems have been studied by researchers for the past three decades, for example, controllability [20], observability [11], and the model reference control problem [19]. A new research area for max-plus linear systems is to establish the geometric control theory [21] as predicted in [4]. There are some existing research results on generalizing fundamental concepts and problems in geometric control to max-plus linear systems, such as computation of different controlled invariant sets ([9], [12], [17]) and the disturbance decoupling problem [14]. This paper reports upon further investigations on the disturbance decoupling problem for max-plus linear systems. Different concepts of the disturbance decoupling problem are introduced, as well as the corresponding solvability conditions and controller synthesis procedures. The main results are illustrated by manufacturing systems.

\section{MAthematical Preliminaries}

A. Residuation, Idempotent Semirings, and Equivalence Kernel

A semiring is a set $\mathcal{S}$, equipped with two operations $\oplus, \otimes$, such that $(\mathcal{S}, \oplus)$ is a commutative monoid (the zero element will be denoted $\varepsilon),(\mathcal{S}, \otimes)$ is a monoid (the unit element will be denoted $e$ ), operation $\otimes$ is right and left distributive over $\oplus$, and $\varepsilon$ is absorbing for the product (i.e. $\varepsilon \otimes a=a \otimes \varepsilon=$ $\varepsilon, \forall a)$. A semiring $\mathcal{S}$ is idempotent if $a \oplus a=a$ for all $a \in \mathcal{S}$. A non empty subset $\mathcal{B}$ of a semiring $\mathcal{S}$ is a subsemiring of $\mathcal{S}$ if for all $a, b \in \mathcal{B}$ we have $a \oplus b \in \mathcal{B}$ and $a \otimes b \in \mathcal{B}$.

Definition 1: Let $\mathcal{S}$ be an idempotent semiring. An order ideal set is a nonempty subset $\mathcal{X}$ of $\mathcal{S}$ such that

$$
(x \in \mathcal{X} \text { and } y \preceq x) \Longrightarrow y \in \mathcal{X} \text {. }
$$

L. Hardouin is a professor in the Laboratorie d'Ingénierie des Systèmes Automatisés of the University of Angers, 62 avenue Notre Dame du Lac, 49000 Angers, France hardouindistia.univ-angers.fr.

M. Lhommeau is an associate professor in the Laboratorie d'Ingénierie des Systèmes Automatisés of the University of Angers, 62 avenue Notre Dame du Lac, 49000 Angers, France lhommeaudistia.univ-angers. fr.

Y. Shang is an assistant professor in the Department of Electrical and Computer Engineering, Southern Illinois University Edwardsville, Campus Box 1801, Edwardsville, IL 62026, USA y shang@siue.edu. This work is supported by 2010-2011 Seed Grants for Transitional and Exploratory Projects (S.T.E.P.) at Southern Illinois University Edwardsville.

Author names are in alphabetically order.
In an idempotent semiring $\mathcal{S}$, operation $\oplus$ induces a partial order relation

$$
a \succeq b \quad \Longleftrightarrow \quad a=a \oplus b, \quad \forall a, b \in \mathcal{S} .
$$

Then, $a \vee b=a \oplus b$. We say that an idempotent semiring $\mathcal{S}$ is complete if it is complete as an ordered set, and if for all $a \in \mathcal{S}$, the left and right multiplications ${ }^{1}$ by $a, L_{a}$ : $\mathcal{S} \rightarrow \mathcal{S}, x \mapsto a x$ and $R_{a}: \mathcal{S} \rightarrow \mathcal{S}, x \mapsto x a$ are lower semicontinuous. These maps are residuated, then the following notation are considered :

$$
\begin{aligned}
& L_{a}^{\sharp}(b)=a \phi b=\bigoplus\{x \mid a x \preceq b\} \text { and } \\
& R_{a}^{\sharp}(b)=b \phi a=\bigoplus\{x \mid x a \preceq b\}, \quad \forall a, b \in \mathcal{S} .
\end{aligned}
$$

Definition 2 (Kernel [2], [3], [5]): Let $\mathcal{S}$ be a complete idempotent semiring and let $C$ be a $n \times p$ matrix with entries in $\mathcal{S}$. We call null kernel of $C$ as the set of elements $x \in \mathcal{S}^{p}$ such that $C x=\epsilon$, denoted as $\operatorname{ker} C$. We call equivalence kernel of $L_{C}$ (denoted by $\operatorname{ker}_{\mathrm{eq}} C$ ), the subset of all pairs of elements of $\mathcal{S}^{p}$ whose components are both mapped by $L_{C}$ to the same element in $\mathcal{S}^{n}$, i.e., the following definition

$$
\operatorname{ker}_{\mathrm{eq}} C:=\left\{\left(s, s^{\prime}\right) \in\left(\mathcal{S}^{p}\right)^{2} \mid C s=C s^{\prime}\right\} .
$$

Clearly $\operatorname{ker}_{\mathrm{eq}} C$, is an equivalence relation on $\mathcal{X}$, i.e., $C s=$ $C s^{\prime} \Longleftrightarrow s^{\prime} \equiv s\left(\bmod \operatorname{ker}_{\mathrm{eq}} C\right)$ and furthermore it is a congruence and then we can define the quotient $\mathcal{S} / \operatorname{ker} C$.

Notation 1: The subset of elements $s^{\prime} \in \mathcal{S}^{p}$ that are equivalent to $s$ modulo $\operatorname{ker} C$ is denoted $[s]_{C}$, i.e.,

$$
[s]_{C}=\left\{s^{\prime} \in \mathcal{S}^{p} \mid s^{\prime} \equiv s\left(\bmod \operatorname{ker}_{\mathrm{eq}} C\right)\right\} \subset \mathcal{S}^{p} .
$$

\section{B. (A, B)-Invariance for Max-Plus Linear Systems}

Max-plus linear systems over the max-plus algebra $\mathbb{R}_{\text {Max }}$, in which the addition $\oplus$ is $\max$ and the multiplication $\otimes$ is + , are described by the following equations:

$$
\begin{aligned}
& x(k)=A x(k-1) \oplus B u(k), \\
& y(k)=C x(k) \oplus D u(k),
\end{aligned}
$$

where $x$ is in the state semimodule $X \cong \mathbb{R}_{\text {Max }}^{n}, y$ is in the output semimodule $Y \cong \mathbb{R}_{\text {Max }}^{q}$, and $u$ is in the input semimodule $U \cong \mathbb{R}_{\text {Max. }}^{p} A: X \rightarrow X, B: U \rightarrow X, C$ : $X \rightarrow Y$ and $D: U \rightarrow Y$ are four $R$-semimodule morphisms.

Given the max-plus linear system (3), a sub-semimodule $\mathcal{V}$ of the state semimodule $X$ is called

- $(A, B)$-invariant, or controlled invariant, if and only if, for all $x_{0} \in \mathcal{V}$, there exists a sequence of control inputs, $\underline{u}=\left\{u_{1}, u_{2}, \cdots\right\}$, such that every component in the state trajectory produced by this input, $\underline{x}\left(x_{0} ; \underline{u}\right)=$ $\left\{x_{0}, x_{1}, \cdots\right\}$, remains inside of $\mathcal{V}$.

- called $(A, B)$-invariant of feedback type if and only if there exists a state feedback $F: X \rightarrow U$ such that $(A \oplus B F) \mathcal{V} \subset \mathcal{V}$.

The family of the controlled invariant sub-semimodules in a sub-semimodule $\mathcal{K}$ of $X$ is closed under the operation $\oplus$. It is a upper semilattice relative to sub-semimodule inclusion $\subset$. Therefore, there exists the supremal element $\mathcal{V}^{*}$ in the

\footnotetext{
${ }^{1}$ The symbol $\otimes$ is often omitted.
} 
family of controlled invariant sub-semimodules in a subsemimodule $\mathcal{K}$ of the state semimodule $X$ and it can be computed by the following algorithm.

Theorem 1: [12] Let $\left\{\mathcal{V}_{k}\right\}_{k \geq 0}$ be the family of subsemimodules defined recursively by

$$
\begin{aligned}
\mathcal{V}_{0} & =\mathcal{K} \\
\mathcal{V}_{k+1} & =\mathcal{V}_{k} \cap A^{-1}\left(\mathcal{V}_{k} \ominus \mathcal{B}\right),
\end{aligned}
$$

where $A^{-1}\left(\mathcal{V}_{k} \ominus \mathcal{B}\right) \triangleq\{x \in X \mid \exists b \in \mathcal{B}$, s.t. $A x \oplus b \in$ $\left.\mathcal{V}_{k}\right\}$, and $\mathcal{B} \triangleq B(U)$. If there exists a nonempty $\cap_{k \in \mathbb{N}} \mathcal{V}_{k}$, then any $(A, B)$-invariant sub-semimodule of $\mathcal{K}$ is contained in $\cap_{k \in \mathbb{N}} \mathcal{V}_{k}$, namely the supremal controlled invariant subsemimodule $\mathcal{V}^{*}$ is also contained in $\cap_{k \in \mathbb{N}} \mathcal{V}_{k}$. Moreover, if the algorithm in Eq. (4) terminates in $r$ steps, then $\mathcal{V}^{*}=\mathcal{V}_{r}$.

\section{Disturbance Decoupling Problem For} MAX-PLUS LINEAR SYSTEMS

A max-plus linear system with disturbances is defined as

$$
\begin{aligned}
x(k) & =A x(k-1) \oplus B u(k) \oplus S q(k), \\
y(k) & =C x(k),
\end{aligned}
$$

where $x(k) \in X \cong \mathbb{R}_{\text {Max }}^{n}, u(k) \in U \cong \mathbb{R}_{\text {Max }}^{p}, q(k) \in Q \cong$ $\mathbb{R}_{\text {Max }}^{r}, y(k) \in Y \cong \mathbb{R}_{\text {Max }}^{\text {Max }}$ and $k \in \mathbb{Z}$.

\section{A. Disturbance Decoupling Problem}

For max-plus linear systems in Eq. (5), we introduce the definition of the disturbance decoupling problem(DDP):

Definition 3: The system (5) is called disturbance decoupled by an open-loop controller $u(k)=v(k)$ (or by a statefeedback controller $u(k)=F x(k-1) \oplus v(k))$ if and only if any disturbance signal will not affect the system output $y(k)$ for all $k \in \mathbb{Z}$ and for any initial condition $x_{0}$.

Theorem 2: [21] The DDP is solvable by a state feedback controller for linear systems over a field if and only if the supremal controlled invariant subpace of feedback type in $\mathcal{K}$ contains $\mathcal{T}$, where $\mathcal{K}$ is the null kernel of $C$ and $\mathcal{T}=\operatorname{Im} S$.

The following proposition shows that the solvability condition for the DDP by a state-feedback controller $u(k)=$ $F x(k-1) \oplus v(k)$, where $v(k)=\epsilon$, of a max-plus linear system described in Eq. (5) is the same as the traditional linear systems over fields.

Proposition 1: The DDP is solvable by a state-feedback controller $u(k)=F x(k-1) \oplus v(k)$, where $v(k)=\epsilon$, for a max-plus linear system of the form (5), if and only if

$$
\langle A \oplus B F \mid \operatorname{Im} S\rangle:=\operatorname{Im}(A \oplus B F)^{*} S \subseteq \operatorname{ker} C,
$$

is satisfied, where $\operatorname{Im}(A \oplus B F)^{*} S=\operatorname{Im} S \oplus \operatorname{Im}(A \oplus B F) S \oplus$ $\cdots \oplus \operatorname{Im}(A \oplus B F)^{n-1} S \oplus \cdots$.

Proof: If the DDP is solvable by a state-feedback controller $u(k)=F x(k-1) \oplus v(k)$, where $v(k)=\epsilon$, then, for any initial condition $x(0)$, the original output signals are the same as the output signals induced by disturbances for all $n$.

$$
\begin{aligned}
y(n) & =y_{q}(n) \Longleftrightarrow C x(n)=C x_{q}(n) \\
x(n) & =(A \oplus B F)^{n} x(0) \\
x_{q}(n) & =(A \oplus B F)^{n} x(0) \oplus\langle A \oplus B F \mid \operatorname{Im} S\rangle_{n},
\end{aligned}
$$

where $\langle A \oplus B F \mid \operatorname{Im} S\rangle_{n}=\operatorname{Im} S \oplus \operatorname{Im}(A \oplus B F) S \oplus \cdots \oplus \operatorname{Im}(A \oplus$ $B F)^{n-1} S$, this equality has to hold for $x(0)=\epsilon$, so it means $\langle A \oplus B F \mid \operatorname{Im} S\rangle_{n}$ is contained in the null kernel of $C$, $\operatorname{ker} C$, for all $n$. Hence, we have $\langle A \oplus B F \mid \operatorname{Im} S\rangle \subseteq \operatorname{Ker} C$. On the other hand, if $\langle A \oplus B F \mid \operatorname{Im} S\rangle \subseteq \operatorname{Ker} C$ is satisfied, then Eq. (7) holds for any $n$ and any initial conditions. Hence, the DDP is solvable by a state-feedback controller.

If the null kernel of $C$ is nontrivial, i.e not the same as $\epsilon$, this result will evolve with the calculation of $(A \oplus B F)$ invariant semimodule in the null kernel of $C$, and then verification of whether the $(A \oplus B F)$-invariant semimodule contains the image of $S$. The computational methods of geometric and algebraic invariant sets have been introduced by Katz in [12].

Proposition 2: Given a max-plus linear system of the form (5), the DDP is solvable by a state feedback controller $u(k)=F x(k-1) \oplus v(k)$ for $v(k) \neq \epsilon$ if and only if there exist a state feedback mapping $F$ and a control sequence $\vec{v}=\left[\begin{array}{llll}v(1) & v(2) & \cdots & v(n)\end{array}\right]^{T}$ such that the equivalence relation holds

\section{$\langle A \oplus B F \mid B\rangle_{n} \vec{v} \oplus\langle A \oplus B F \mid S\rangle_{n} \vec{q} \equiv_{\text {kereq }_{\text {e }} C}\langle A \oplus B F \mid B\rangle_{n} \vec{v}$}

for all $n$ and any disturbance signal $\vec{q}=$ $\left[\begin{array}{llll}q(1) & q(2) & \cdots & q(n)\end{array}\right]^{T}$, where $\langle A \oplus B F \mid S\rangle_{n}=$

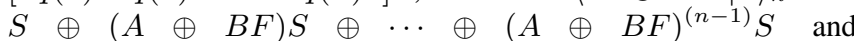
$\langle A \oplus B F \mid B\rangle_{n}=B \oplus(A \oplus B F) B \oplus \cdots \oplus(A \oplus B F)^{(n-1)} B$.

Proof: If the DDP is solvable by a state-feedback controller $u(k)=F x(k-1) \oplus v(k)$, then, for any initial condition $x(0)$, we require that the original output signals are the same as the output signals induced by disturbances for all $n$, that is,

$$
\begin{aligned}
y(n)= & y_{q}(n) \Longleftrightarrow C x(n)=C x_{q}(n) \\
x(n)= & (A \oplus B F)^{n} x(0) \oplus\langle(A \oplus B F) \mid B\rangle_{n} \vec{v} \\
x_{q}(n)= & (A \oplus B F)^{n} x(0) \oplus\langle A \oplus B F \mid B\rangle_{n} \vec{v} \\
& \oplus\langle A \oplus B F \mid S\rangle_{n} \vec{q} .
\end{aligned}
$$

This equality has to hold for $x(0)=\epsilon$, it means that the equivalence relation holds

$$
\langle A \oplus B F \mid B\rangle_{n} \vec{v} \oplus\langle A \oplus B F \mid S\rangle_{n} \vec{q} \equiv_{\text {kereq }_{\text {eq }} C}\langle A \oplus B F \mid B\rangle_{n} \vec{v}
$$

for all $n$ and any disturbance signal $\vec{q}$. On the other hand, if the equation holds for all $n$, we have $y(n)=y_{q}(n)$ for any initial conditions.

Proposition 3: Given a max-plus linear system of the form (5), the DDP is solvable by an open-loop controller $u(k)=v(k)$ if and only if there exists an open-loop control sequence $\vec{v}=\left[\begin{array}{llll}v(1) & v(2) & \cdots & v(n)\end{array}\right]^{T}$ such that the equivalence relation holds

$$
\langle A \mid B\rangle_{n} \vec{v} \oplus\langle A \mid S\rangle_{n} \vec{q} \equiv_{\operatorname{ker}_{\text {eq }} C}\langle A \mid B\rangle_{n} \vec{v}
$$

for all $n$ and any disturbance signal $\vec{q}=$ $\left[\begin{array}{llll}q(1) & q(2) & \cdots & q(n)\end{array}\right]^{T}$, where $\langle A \mid S\rangle_{n}=S \oplus A S \oplus$ $\cdots \oplus A^{(n-1)} S$ and $\langle A \mid B\rangle_{n}=B \oplus A B \oplus \cdots \oplus A^{(n-1)} B$.

Proof: If the DDP is solvable by an open-loop controller $u(k)=v(k)$, then, for any initial condition $x(0)$, we require that the original output signals are the same as the output signals induced by disturbances for all $n$, that is,

$$
\begin{aligned}
y(n) & =y_{q}(n) \Longleftrightarrow C x(n)=C x_{q}(n) \\
x(n) & =A^{n} x(0) \oplus\langle A \mid B\rangle_{n} \vec{v} \\
x_{q}(n) & =A^{n} x(0) \oplus\langle A \mid B\rangle_{n} \vec{v} \oplus\langle A \mid S\rangle_{n} \vec{q} .
\end{aligned}
$$

This equality has to hold for $x(0)=\epsilon$, it means that the equivalence relation holds

$$
\langle A \mid B\rangle_{n} \vec{v} \oplus\langle A \mid S\rangle_{n} \vec{q} \equiv_{\operatorname{ker}_{\text {eq }} C}\langle A \mid B\rangle_{n} \vec{v}
$$

for all $n$ and any disturbance signal $\vec{q}$. On the other hand, if the equation holds for all $n$, we have $y(n)=y_{q}(n)$ for any initial conditions.

Therefore, the solvability condition of the DDP by an open-loop controller needs an infinite checking of the equivalence relations induced by the output mapping $C$. An alternative method will be presented in the next section using frequency domain representations. 


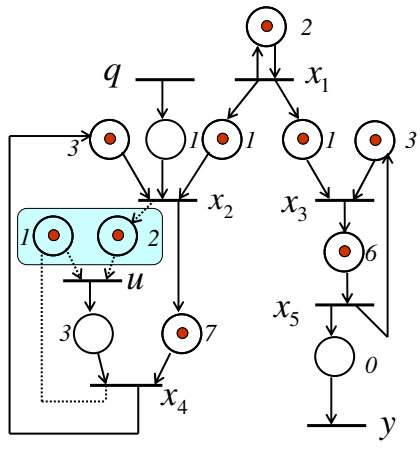

Fig. 1: The timed Petri net for a queueing system.

\section{B. Example}

Given a simple queueing system modelled by a timed Petri net as shown in Fig. 1. If we assume the output is the customer arrival time of the second server, then we can write the system equation as a max-plus linear system in Eq. (5) with the system matrices as

$$
A=\left[\begin{array}{lllll}
2 & \epsilon & \epsilon & \epsilon & \epsilon \\
1 & \epsilon & \epsilon & 3 & \epsilon \\
1 & \epsilon & \epsilon & \epsilon & 3 \\
\epsilon & 7 & \epsilon & \epsilon & \epsilon \\
\epsilon & \epsilon & 6 & \epsilon & \epsilon
\end{array}\right], B=\left[\begin{array}{c}
\epsilon \\
\epsilon \\
\epsilon \\
3 \\
\epsilon
\end{array}\right] \quad S=\left[\begin{array}{c}
\epsilon \\
1 \\
\epsilon \\
\epsilon \\
\epsilon
\end{array}\right] \text { and }
$$

$C=\left[\begin{array}{lllll}\epsilon & \epsilon & \epsilon & \epsilon & e\end{array}\right]$. The null kernel of $C$ is generated by the four basis vectors $\mathbf{e}_{\mathbf{1}}, \mathbf{e}_{\mathbf{2}}, \mathbf{e}_{\mathbf{3}}$, and $\mathbf{e}_{\mathbf{4}}$, where $\mathbf{e}_{\mathbf{1}}=$ $[e, \epsilon, \epsilon, \epsilon, \epsilon]^{T}, \mathbf{e}_{2}=[\epsilon, e, \epsilon, \epsilon, \epsilon]^{T}, \mathbf{e}_{\mathbf{3}}=[\epsilon, \epsilon, e, \epsilon, \epsilon]^{T}$, and $\mathbf{e}_{\mathbf{4}}=[\epsilon, \epsilon, \epsilon, e, \epsilon]^{T}$. In order to calculate the supremal $(A, B)$ invariant sub-semimodule $\mathcal{V}^{*}$ in the null kernel of $C$, we can either use the algorithm in Eq. (4) to obtain that

$$
\begin{aligned}
\mathcal{V}_{0} & =\mathcal{K}=\operatorname{Ker} C \\
\mathcal{V}_{1} & =V_{0} \cap A^{-1}\left(\mathcal{V}_{0} \ominus \mathcal{B}\right)=\operatorname{Span}\left[\mathbf{e}_{1}, \mathbf{e}_{2}, \mathbf{e}_{4}\right] \\
\mathcal{V}_{2} & =V_{1} \cap A^{-1}\left(\mathcal{V}_{1} \ominus \mathcal{B}\right)=\operatorname{Span}\left[\mathbf{e}_{2}, \mathbf{e}_{4}\right] \\
& \vdots \\
\mathcal{V}_{k} & =\operatorname{Span}\left[\mathbf{e}_{2}, \mathbf{e}_{4}\right]=\mathcal{V}^{*} .
\end{aligned}
$$

Because the $(A, B)$-invariant semimodule is not identical with $(A \oplus B F)$-invariant semimodule, we need to verify for any point $x$ in $\mathcal{V}^{*}$, whether there exists a state feedback $F: X \rightarrow U$ such that $\mathcal{V}^{*}$ is $(A \oplus B F)$-invariant. If assume $F=\left[f_{1}, f_{2}, f_{3}, f_{4}, f_{5}\right]$ for any $f_{i} \in \mathbb{R}_{\text {Max }}$ and any $x=\left[\epsilon, x_{2}, \epsilon, x_{4}, \epsilon\right]$ in $\mathcal{V}^{*}$, one can pick any values for $F$ such that $\mathcal{V}^{*}$ is $(A \oplus B F)$-invariant. We chose $F=[\epsilon, 2, \epsilon, 1, \epsilon]$ and the state feedback controller is shown in the shaded box shown in Fig. 1, the $k$-th processing time of $u(k)$ is the maximum of $2+x_{2}(k-1)$ and $1+x_{4}(k-1)$. The image of $S$ is contained in the supremal $(A \oplus B F)$-invariant sub-semimodule of the null kernel of $C$, therefore, the DDP is solvable by a state-feedback controller $u(k)=F x(k-1) \oplus v(k)$, with $v(k)=\epsilon$.

Remark 1: We need to notice that the output signal is completely decoupled from the disturbance signal in this example, therefore, the DDP is solvable with or without controller. If the output signals include $x_{4}$ and $x_{5}$, then the solvability condition for the DDP for a state-space controller $u(k)=F x(k-1) \oplus v(k)$, with $v(k)=\epsilon$, is not satisfied. In other word, if a delay or breakdown has already occurred in a discrete-event system, one cannot remove the delay or breakdown using any controller. Even when we consider the state feedback controller $u(k)=F x(k-1) \oplus v(k)$ with $v(k) \neq \epsilon$, Proposition 2 implies that we have to delay the process same as the disturbance has placed on the system or even more than that. Therefore, the traditional definition
DDP is very restrictive for max-plus linear systems, a modified DDP with better practical meanings will be introduced in the following section.

\section{MOdified DDP FOR MAX-Plus Linear Systems}

In the max-plus linear system described in Eq. (5), the traditional null kernel of a nontrivial matrix $C$ is trivial. This will conclude that $\operatorname{Im} S$ is $\epsilon$. This argument means that for a nontrivial disturbance matrix $S$, one cannot find a state-feedback controller such that the output will not be affected by the disturbance. From the practical point of view, a modified DDP for max-plus linear systems is defined as follows:

Definition 4: The max-plus linear system described in Eq. (5) is called modified disturbance decoupled by an openloop controller $u(k)=v(k)$ (or by a closed-loop controller $u(k)=F x(k-1) \oplus v(k))$ if and only if the system output signals will not be disturbed more than the disturbances have.

\section{A. Solving Modified DDP using Frequency Domain Repre- sentation}

A trajectory of a timed event graph transition $x$ is a firing date sequence $\{x(k)\} \in \mathbb{Z}$. For each increasing sequence $\{x(k)\}$, it is possible to define the transformation $X(\gamma)=$ $\bigoplus x(k) \gamma^{k}$ where $\gamma$ is a backward shift operator in event $k \in \mathbb{Z}$

domain (i.e., $Y(\gamma)=\gamma X(\gamma) \Longleftrightarrow\{y(k)\}=\{x(k-1)\}$, (see [1], p. 228). This transformation is analogous to the $Z$ transform used in discrete-time classical control theory and the formal series $X(\gamma)$ is a synthetic representation of the trajectory $x(k)$. The set of the formal power series in $\gamma$ is denoted by $\overline{\mathbb{Z}}_{\max } \llbracket \gamma \rrbracket$ and constitutes an idempotent semiring. Therefore, the state equation in Eq. (5) becomes a polynomial equation or a frequency domain representation,

$$
\begin{aligned}
& X(\gamma)=\gamma A X(\gamma) \oplus x_{0} \oplus B U(\gamma) \oplus S Q(\gamma) \\
& Y(\gamma)=C X(\gamma)
\end{aligned}
$$

for any initial state $x_{0}$, assuming $u(0)=q(0)=\epsilon$, the state $X(\gamma) \in \mathcal{X}=\left(\overline{\mathbb{Z}}_{\max } \llbracket \gamma \rrbracket\right)^{n \times 1}$, the output $Y(\gamma) \in \mathcal{Y}=$ $\left(\overline{\mathbb{Z}}_{\max } \llbracket \gamma \rrbracket\right)^{q \times 1}$, the input $U(\gamma) \in \mathcal{U}=\left(\overline{\mathbb{Z}}_{\max } \llbracket \gamma \rrbracket\right)^{p \times 1}$, and the disturbance $Q(\gamma) \in \mathcal{Q}=\left(\overline{\mathbb{Z}}_{\max } \llbracket \gamma \rrbracket\right)^{r \times 1}$.

If we assume the initial state is $\epsilon$, then the model equation is given by

$$
\begin{aligned}
& X(\gamma)=\bar{A} X(\gamma) \oplus B U(\gamma) \oplus S Q(\gamma), \text { where } \bar{A}=\gamma A, \\
& Y(\gamma)=C X(\gamma) .
\end{aligned}
$$

Matrices $\bar{A} \in\left(\overline{\mathbb{Z}}_{\max } \llbracket \gamma \rrbracket\right)^{n \times n}, B \in\left(\overline{\mathbb{Z}}_{\max } \llbracket \gamma \rrbracket\right)^{n \times p}, C \in$ $\left(\overline{\mathbb{Z}}_{\max } \llbracket \gamma \rrbracket\right)^{q \times n}$ and $S \in\left(\overline{\mathbb{Z}}_{\max } \llbracket \gamma \rrbracket\right)^{n \times r}$ represent the link between transitions. The trajectories $U(\gamma)$ and $Y(\gamma)$ can be related ([1], p. 243) by the equation $Y(\gamma)=H(\gamma) U(\gamma)$, where $H(\gamma)=C \bar{A}^{*} B \in\left(\overline{\mathbb{Z}}_{\max } \llbracket \gamma \rrbracket\right)^{q \times p}$ is called the transfer matrix of the TEG. Entries of matrix $H$ are periodic series ([1], p. 260) in the idempotent semiring, usually represented by $p(\gamma) \oplus q(\gamma)\left(\tau \gamma^{\nu}\right)^{*}$, where $p(\gamma)$ is a polynomial representing the transient behavior, $q(\gamma)$ is a polynomial corresponding to a pattern which is repeated periodically, the period being given by the monomial $\left(\tau \gamma^{\nu}\right)$.

\section{B. Modified DDP with an Open-Loop Controller}

The control of a transition means that the firing may be enable or disable, that means, the input date is controlled. Therefore, a control law aims to control the input date of tokens in order to achieve some specifications. A classical specification is to track a trajectory (a reference output sequence) while delaying as much as possible the token input, this strategy consists in computing the optimal control with regard to the well-known just-in-time criterion. 
Formally, let $Z(\gamma) \in \overline{\mathbb{Z}}_{\max } \llbracket \gamma \rrbracket^{q}$ be a given reference output, the problem is to compute the greatest control, denoted $U_{\text {opt }}(\gamma) \in \overline{\mathbb{Z}}_{\max } \llbracket \gamma \rrbracket^{p}$ such that $Y(\gamma) \preceq Z(\gamma)$. Among the controls which respect the constraint $Y(\gamma) \preceq Z(\gamma)$, $U_{o p t}(\gamma)$ is the greatest, i.e., the one which delays as much as possible the input of the tokens in the graph, i.e., this control minimizes in an optimal manner the sojourn time of tokens.

To formalize the preceding arguments, the objective of the modified DDP using an open-loop controller is to find an open-loop controller $U(\gamma)$ such that, for any initial condition $x_{0}$ in the state space, the system output will not be disturbed more than the disturbance signal has, i.e. the following equation holds for any initial condition $x_{0}$ :

$$
\begin{aligned}
X(\gamma) & =\bar{A}^{*} B U(\gamma) \oplus \bar{A}^{*} S Q(\gamma) \oplus \bar{A}^{*} x_{0} \\
& =\bar{A}^{*}[B \mid S]\left(\begin{array}{l}
U(\gamma) \\
Q(\gamma)
\end{array}\right) \oplus \bar{A}^{*} x_{0} \\
& =\bar{A}^{*} \widetilde{B}\left(\begin{array}{l}
U(\gamma) \\
Q(\gamma)
\end{array}\right) \oplus \bar{A}^{*} x_{0} \\
Y(\gamma) & =C \bar{A}^{*} B U(\gamma) \oplus C \bar{A}^{*} S Q(\gamma) \oplus C \bar{A}^{*} x_{0} \\
& =C \bar{A}^{*} \widetilde{B}\left(\begin{array}{l}
U(\gamma) \\
Q(\gamma)
\end{array}\right) \oplus C \bar{A}^{*} x_{0} .
\end{aligned}
$$

In order to solve for the modified DDP using an openloop controler, then according to Definition 4, the following equality has to hold for any initial condition:

$$
\begin{aligned}
C \bar{A}^{*} \widetilde{B}\left(\begin{array}{l}
U(\gamma) \\
Q(\gamma)
\end{array}\right) \oplus C \bar{A}^{*} x_{0} & =C \bar{A}^{*} S Q(\gamma) \oplus C \bar{A}^{*} x_{0} \Longleftrightarrow \\
C \bar{A}^{*} \widetilde{B}\left(\begin{array}{l}
U(\gamma) \\
Q(\gamma)
\end{array}\right) & =C \bar{A}^{*} S Q(\gamma), \forall x_{0}
\end{aligned}
$$

In other words, the objective of open-loop controller DDP is to characterize the greatest state $X(\gamma) \in \operatorname{Im} \bar{A}^{*} \widetilde{B}^{2}=$

$$
\begin{gathered}
\left\{\bar{A}^{*} \widetilde{B}\left(\begin{array}{l}
U(\gamma) \\
Q(\gamma)
\end{array}\right) \mid(U(\gamma), Q(\gamma)) \in \mathcal{U} \times \mathcal{Q}\right\} \text { such that } \\
C X(\gamma)=C \bar{A}^{*} S Q(\gamma), \forall Q(\gamma) \in \mathcal{Q} \Longleftrightarrow \\
\left(X(\gamma), \bar{A}^{*} S Q(\gamma)\right) \in \operatorname{ker}_{\text {eq }} C, \forall Q(\gamma) \in \mathcal{Q}
\end{gathered}
$$

This is the greatest state $X(\gamma) \in \operatorname{Im} \bar{A}^{*} \widetilde{B}$ ensuring that the output $Y(\gamma)$ is equal to the one due to the disturbances. Because we modified the DDP, the solvability of the DDP is not an issue anymore because there is always a minimal solution $U(\gamma)=\epsilon$ to the equality. Moreover, the equation in (16) is also equivalent as

$$
\begin{aligned}
& C \bar{A}^{*} B U(\gamma) \preceq C \bar{A}^{*} S Q(\gamma) \Longleftrightarrow \\
& U(\gamma) \preceq\left(C \bar{A}^{*} B\right) \phi\left(C \bar{A}^{*} S Q(\gamma)\right)=U(\gamma)_{o p t} .
\end{aligned}
$$

Therefore, $U(\gamma)_{\text {opt }}$ is the optimal solution to solve the modified DDP for max-plus linear systems. We need to notice that if the condition $\operatorname{Im} C \bar{A}^{*} S \subset \operatorname{Im} C \bar{A}^{*} B$ holds, i.e., if $\exists L$ such that $C \bar{A}^{*} S=C \bar{A}^{*} B L$ then the optimal solution solution $U(\gamma)_{\text {opt }}$ becomes the solution to the equality

$$
\begin{aligned}
& C \bar{A}^{*} B U(\gamma)_{o p t}=C \bar{A}^{*} B\left(\left(C \bar{A}^{*} B\right) \phi\left(C \bar{A}^{*} B L Q(\gamma)\right)\right. \\
& =C \bar{A}^{*} B L Q(\gamma)=C \bar{A}^{*} S Q(\gamma) \Longrightarrow \\
& C \bar{A}^{*} B U(\gamma)_{o p t} \oplus C \bar{A}^{*} S Q(\gamma)=C \bar{A}^{*} B U(\gamma)_{o p t} .
\end{aligned}
$$

The equality in (18) means that the same optimal controller $U(\gamma)_{\text {opt }}$ can also solve the DDP in Definition 3 with an openloop controller, which means finding an open-loop controller such that the disturbance will not affect the system output. Moreover, the infinite checking of the equivalence relation in Eq. (8) is reduced to one checking for the polynomial equation. Even if the equality is not achieved by the control signal $U(\gamma)_{o p t}$, the solution still has sense because it is the greatest one which ensures to be as close as the possible the solution such that output $Y(\gamma)$ is unchanged.

\section{Modified DDP with a Closed-Loop Controller}

In this paper a specific design goal is to compute a closedloop controller $F$ (i.e., $u(k)=F x(k-1) \oplus v(k)$ ) in order to take into account the influence of the uncontrollable input $q$. An uncontrollable input $q_{i}$ may disable the firing of the internal transitions bind to $q_{i}$ through matrix $S$. Therefore, this uncontrollable input $q_{i}$ may decreased the performance of the system, i.e., the token output may be delayed, and some tokens may needlessly wait in the graph since the system is blocked. Therefore, the controller design aims to obtain the greatest $F$ which avoid the input of useless tokens. This means that controller $F$ must be the greatest such that the output $y$, (i.e., with the control $u(k)=F x(k-1) \oplus v(k)$ ) be equal to the output without controller (i.e., with $u(k)=$ $v(k))$, in other words the control must be neutral with regard to the output, i.e., it must not disturb the system more than disturbance $q$ does it. From the just-in-time point of view it is the best that we can do.

In [7], [18] closed-loop controllers synthesis, in order to achieve the model matching problem, is addressed. The objective is to compute the greatest closed-loop controller $\bar{F}=\gamma F \in\left(\overline{\mathbb{Z}}_{\max } \llbracket \gamma \rrbracket\right)^{p \times n}$ (with $U(\gamma)=\gamma F X(\gamma) \oplus V(\gamma)$ ), which ensures that output $Y(\gamma) \preceq G_{\text {ref }} V(\gamma)$, where $G_{\text {ref }} \in$ $\left(\overline{\mathbb{Z}}_{\max } \llbracket \gamma \rrbracket\right)^{q \times p}$ is a model to track. This controller leads to an exact model matching if possible and delays as much as possible the input of token while ensuring the constraint $\left(Y(\gamma) \preceq G_{r e f} V(\gamma)\right)$. If $U(\gamma)=\gamma F X(\gamma) \oplus V(\gamma):=$ $\bar{F} X(\gamma) \oplus V(\gamma)$, then the system equation in Eq. (11) becomes

$$
\begin{aligned}
X(\gamma)= & {[\gamma(A \oplus B F)] X(\gamma) \oplus x_{0} \oplus B V(\gamma) \oplus S Q(\gamma) } \\
= & (\bar{A} \oplus B \bar{F}) X(\gamma) \oplus x_{0} \oplus B V(\gamma) \oplus S Q(\gamma) \\
= & (\bar{A} \oplus B \bar{F})^{*}[B \mid S]\left(\begin{array}{l}
V(\gamma) \\
Q(\gamma)
\end{array}\right) \oplus(\bar{A} \oplus B \bar{F})^{*} x_{0} \\
= & (\bar{A} \oplus B \bar{F})^{*} \widetilde{B}\left(\begin{array}{l}
V(\gamma) \\
Q(\gamma)
\end{array}\right) \oplus(\bar{A} \oplus B \bar{F})^{*} x_{0} \\
Y(\gamma)= & C X(\gamma)=C(\bar{A} \oplus B \bar{F})^{*} \widetilde{B}\left(\begin{array}{l}
V(\gamma) \\
Q(\gamma)
\end{array}\right) \\
& \oplus C(\bar{A} \oplus B \bar{F})^{*} x_{0}
\end{aligned}
$$

where $\widetilde{B}=[B \mid S]$. The objective of the modified DDP using a state feedback controller is to find $U(\gamma)=\bar{F} X(\gamma) \oplus$ $V(\gamma)$ such that the output signals are the same as the output signals due to the open-loop controller $U(\gamma)=V(\gamma)$, and the same as the output signals only due to disturbances as well. In summary, that is, the following equality holds

$$
\begin{array}{r}
C(\bar{A} \oplus B \bar{F})^{*} \widetilde{B}\left(\begin{array}{l}
V(\gamma) \\
Q(\gamma)
\end{array}\right) \oplus C(\bar{A} \oplus B \bar{F})^{*} x_{0} \\
=C \bar{A}^{*} \widetilde{B}\left(\begin{array}{l}
V(\gamma) \\
Q(\gamma)
\end{array}\right) \oplus C \bar{A}^{*} x_{0} \\
=C \bar{A}^{*} B V(\gamma) \oplus C \bar{A}^{*} S Q(\gamma) \oplus C \bar{A}^{*} x_{0},
\end{array}
$$

which can be written as :

$$
C(\bar{A} \oplus B \bar{F})^{*} \bar{B}\left(\begin{array}{c}
V(\gamma) \\
Q(\gamma) \\
x_{0}
\end{array}\right)=C \bar{A}^{*} \bar{B}\left(\begin{array}{c}
V(\gamma) \\
Q(\gamma) \\
x_{0},
\end{array}\right)
$$

where $\bar{B}=[B|S| I d]$, and $I d$ denotes the identity matrix. This equality has to hold for any initial condition $x_{0} \in X(\gamma)$, any disturbance input $Q(\gamma)$ and any control $V(\gamma)$. Hence it is equivalent to

$$
\begin{gathered}
C(\bar{A} \oplus B \bar{F})^{*} \bar{B}=C \bar{A}^{*} \bar{B} \Longleftrightarrow \\
\left((\bar{A} \oplus B \bar{F})^{*} \bar{B}, \bar{A}^{*} \bar{B}\right) \in \mathrm{ker}_{\mathrm{eq}} C .
\end{gathered}
$$


The right side of the equivalence shows that $\bar{F}$ must be such that the transfer between state $X(\gamma)$ and control input $\left(\begin{array}{lll}V(\gamma) & Q(\gamma) & x_{0}\end{array}\right)^{t}$ be equivalent to $\bar{A}^{*} \bar{B}$ modulo ker $_{\text {eq }} C$.

Proposition 4: ([15], [16]) The greatest controller $\bar{F}$ such that, $(\bar{A} \oplus B \bar{F})^{*} \bar{B} \in\left[\bar{A}^{*} \bar{B}\right]_{C}$ is given by

$$
\bar{F}=C \bar{A}^{*} B \nmid C \bar{A}^{*} \bar{B} \phi \bar{A}^{*} \bar{B} .
$$

Remark 2: Controller $\bar{F}$ is the greatest such that

$$
(\bar{A} \oplus B \bar{F})^{*} \bar{B} \in \operatorname{Im} \bar{A}^{*} \bar{B} \cap\left[\bar{A}^{*} \bar{B}\right]_{C},
$$

where $\operatorname{Im} \bar{A}^{*} \bar{B}=\left\{\bar{A}^{*} \bar{B}\left[V(\gamma) Q(\gamma) x_{0}\right]^{t}\right\}$ where $V(\gamma) \in$ $\overline{\mathbb{Z}}_{\max } \llbracket \gamma \rrbracket^{p}$ and $Q(\gamma) \in \overline{\mathbb{Z}}_{\max } \llbracket \gamma \rrbracket^{r}$. Indeed, it is sufficient to note that, using the properties of star operations, $(a \oplus$ $b)^{*}=\left(a^{*} b\right)^{*} a^{*}$ and $(a b)^{*} a=a(b a)^{*}$, we have $(\bar{A} \oplus$ $B \bar{F})^{*} \bar{B}=\left(\bar{A}^{*} \bar{B}\left(\begin{array}{l}e \\ \varepsilon \\ \varepsilon\end{array}\right) \bar{F}\right)^{*} \bar{A}^{*} \bar{B}=\bar{A}^{*} \bar{B}\left(\left(\begin{array}{l}e \\ \varepsilon \\ \varepsilon\end{array}\right) \bar{F}^{*} \bar{A}^{*}\right)^{*}$, clearly $(\bar{A} \oplus B \bar{F}) * \bar{B} \in \operatorname{Im} \bar{A} * \bar{B}$.

\section{Application to a Manufacturing System}

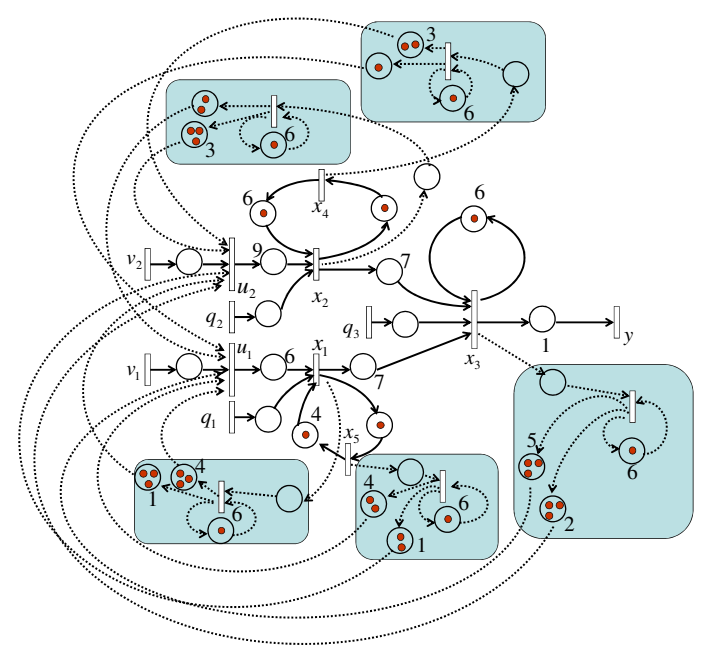

Fig. 2: System in bold line and controller in dotted line (shaded boxes).

A manufacturing system is illustrated in Fig. 2. Transitions $q_{1}, q_{2}$ and $q_{3}$ are uncontrollable inputs (disturbances), which delay the parts output of machines. In a manufacturing context, inputs $q$ may represent machine breakdowns, uncontrollable supplies of raw materials. The system equation can be written as an implicit max-plus linear equation:

$$
\begin{aligned}
x(k) & =A_{0} x(k) \oplus A_{1} x(k-1) \oplus B_{0} u(k) \oplus S_{0} q(k), \\
y(k) & =C x(k),
\end{aligned}
$$

where the system matrices are $A_{0}=\left[\begin{array}{ccccc}\epsilon & \epsilon & \epsilon & \epsilon & \epsilon \\ \epsilon & \epsilon & \epsilon & \epsilon & \epsilon \\ 7 & 7 & \epsilon & \epsilon & \epsilon \\ \epsilon & \epsilon & \epsilon & \epsilon & \epsilon \\ \epsilon & \epsilon & \epsilon & \epsilon & \epsilon\end{array}\right]$, $A_{1}=\left[\begin{array}{lllll}\epsilon & \epsilon & \epsilon & \epsilon & 4 \\ \epsilon & \epsilon & \epsilon & 6 & \epsilon \\ \epsilon & \epsilon & 6 & \epsilon & \epsilon \\ \epsilon & e & \epsilon & \epsilon & \epsilon \\ e & \epsilon & \epsilon & \epsilon & \epsilon\end{array}\right], B_{0}=\left[\begin{array}{cc}6 & \epsilon \\ \epsilon & 9 \\ \epsilon & \epsilon \\ \epsilon & \epsilon \\ \epsilon & \epsilon\end{array}\right], \quad S_{0}=$ $\left[\begin{array}{lll}e & \epsilon & \epsilon \\ \epsilon & e & \epsilon \\ \epsilon & \epsilon & e \\ \epsilon & \epsilon & \epsilon \\ \epsilon & \epsilon & \epsilon\end{array}\right]$, and $C=\left[\begin{array}{lllll}\epsilon & \epsilon & 1 & \epsilon & \epsilon\end{array}\right]$. We can rewrite the system equation into the max-plus linear system in

Eq. (5) where the system matrices are $A=A_{0}^{*} A_{1}=$ $\left[\begin{array}{ccccc}\epsilon & \epsilon & \epsilon & \epsilon & 4 \\ \epsilon & \epsilon & \epsilon & 6 & \epsilon \\ \epsilon & \epsilon & 6 & 13 & 11 \\ \epsilon & e & \epsilon & \epsilon & \epsilon \\ e & \epsilon & \epsilon & \epsilon & \epsilon\end{array}\right], B=A_{0}^{*} B_{0}=\left[\begin{array}{cc}6 & \epsilon \\ \epsilon & 9 \\ 13 & 16 \\ \epsilon & \epsilon \\ \epsilon & \epsilon\end{array}\right]$, and
$S=A_{0}^{*} S_{0}=\left[\begin{array}{ccc}\epsilon & e & \epsilon \\ 7 & 7 & e \\ \epsilon & \epsilon & \epsilon \\ \epsilon & \epsilon & \epsilon\end{array}\right]$.

The frequency domain representation in (12) has matrix $\bar{A}=\gamma A$ for any initial condition $x_{0}$. The transfer function between the output $Y(\gamma)$ and disturbance $Q(\gamma)$ and the input $U(\gamma)$, respectively, are

$$
\begin{aligned}
& C \bar{A}^{*} S=\left(8(6 \gamma)^{*} \quad 8(6 \gamma)^{*} \quad 1(6 \gamma)^{*}\right), \\
& C \bar{A}^{*} B=\left(14(6 \gamma)^{*} \quad 17(6 \gamma)^{*}\right) \text {, }
\end{aligned}
$$

in which each component of these matrices is a periodic series. The example has been computed by using toolbox MinMaxGD, a C++ library allowing to handle periodic series as introduced in section IV.A, it can be noted that this library is also interfaced with Scilab and MATLAB([6]).

According to Proposition 4 and solution (24), the controller is obtained by computing $C \bar{A}^{*} B \phi C \bar{A}^{*} \bar{B}_{\phi} \bar{A}^{*} \bar{B}$, where $B=B$. Therefore, we obtain $\bar{F}=C \bar{A}^{*} B \phi C \bar{A}^{*} \bar{B} \phi \bar{A}^{*} \bar{B}=$

$$
\left(\begin{array}{c}
-2 \gamma^{2}(6 \gamma)^{*} \gamma^{2}(6 \gamma)^{*}-13(6 \gamma)^{*} \gamma(6 \gamma)^{*}-2 \gamma(6 \gamma)^{*} \\
-5 \gamma^{2}(6 \gamma)^{*}-3 \gamma^{2}(6 \gamma)^{*}-16(6 \gamma)^{*}-3 \gamma(6 \gamma)^{*}-5 \gamma(6 \gamma)^{*}
\end{array}\right)
$$

This feedback is not causal because there are negative coefficients in the matrix, The canonical injection from the causal elements of $\overline{\mathbb{Z}}_{\max } \llbracket \gamma \rrbracket$ (denoted $\overline{\mathbb{Z}}_{\max } \llbracket \gamma \rrbracket^{+}$) in $\overline{\mathbb{Z}}_{\max } \llbracket \gamma \rrbracket$ is also residuated (see [7] for details). Its residual is given by $\operatorname{Pr}\left(\bigoplus_{k \in \mathbb{Z}} s(k) \gamma^{k}\right)=\bigoplus_{k \in \mathbb{Z}} s_{+}(k) \gamma^{k}$ where

$$
s_{+}(k)=\left\{\begin{array}{l}
s(k) \text { if }(k, s(k)) \geq(0,0), \\
\varepsilon \text { otherwise. }
\end{array}\right.
$$

Therefore, the greatest causal feedback is $\bar{F}_{+}=\operatorname{Pr}(\bar{F})=$

$$
\left(\begin{array}{ccccc}
4 \gamma^{3}(6 \gamma)^{*} & \gamma^{2}(6 \gamma)^{*} & 5 \gamma^{3}(6 \gamma)^{*} & \gamma(6 \gamma)^{*} & 4 \gamma^{2}(6 \gamma)^{*} \\
1 \gamma^{3}(6 \gamma)^{*} & 3 \gamma^{3}(6 \gamma)^{*} & 2 \gamma^{3}(6 \gamma)^{*} & 3 \gamma^{2}(6 \gamma)^{*} & 1 \gamma^{2}(6 \gamma)^{*}
\end{array}\right)
$$

Figure 2 shows a realization of the controller (bold dotted lines). The greatest causal feedback controller can solve the modified DDP for any initial conditions. In order to simulate the system, we assume for an initial condition $x_{0}=\epsilon$ and the following input $V(\gamma)$ is considered

$$
V(\gamma)=\left(\begin{array}{l}
20 \oplus+\infty \gamma^{6} \\
20 \oplus+\infty \gamma^{6}
\end{array}\right)
$$

It means that 6 tokens are available at time $t=$ 20. First the system is assumed to be not disturbed, i.e., $Q(\gamma)=\varepsilon$. The system trajectories without controller $(\bar{F}=\varepsilon$, then $U(\gamma)=V(\gamma)$, i.e., the openloop behavior), denoted $U_{o l}, X_{o l}$ and $Y_{o l}$, are given by $U_{o l}=V$

$$
\begin{aligned}
X_{o l}= & \bar{A}^{*} B V \\
= & \left(\begin{array}{c}
26 \oplus 30 \gamma^{2} \oplus 34 \gamma^{4} \oplus+\infty \gamma^{6} \\
29 \oplus 35 \gamma^{2} \oplus 41 \gamma^{4} \oplus+\infty \gamma^{6} \\
36 \oplus 42 \gamma \oplus 48 \gamma^{2} \oplus 54 \gamma^{3} \oplus 60 \gamma^{4} \oplus 66 \gamma^{5} \\
\left(\text { cont }^{\prime} \mathrm{d} .\right) \oplus+\infty \gamma^{6} \\
29 \gamma \oplus 35 \gamma^{3} \oplus 41 \gamma^{5} \oplus+\infty \gamma^{7} \\
26 \gamma \oplus 30 \gamma^{3} \oplus 34 \gamma^{5} \oplus+\infty \gamma^{7}
\end{array}\right), \\
Y_{o l}= & C \bar{A}^{*} B V \\
= & 37 \oplus 43 \gamma \oplus 49 \gamma^{2} \oplus 55 \gamma^{3} \oplus 61 \gamma^{4} \oplus 67 \gamma^{5} \oplus+\infty \gamma^{6} .
\end{aligned}
$$


With controller $\bar{F}_{+}$(i.e. $\left.U=\bar{F}_{+} X \oplus V\right)$ these trajectories, denoted $U_{c l}, X_{c l}$ and $Y_{c l}$, become

$$
\begin{aligned}
& U_{c l}=\bar{F}_{+} X_{c l} \oplus V \\
& =\left(\begin{array}{c}
20 \oplus 29 \gamma^{2} \oplus 41 \gamma^{3} \oplus 47 \gamma^{4} \oplus 53 \gamma^{5} \oplus+\infty \gamma^{6} \\
20 \oplus 38 \gamma^{3} \oplus 44 \gamma^{4} \oplus 50 \gamma^{5} \oplus+\infty \gamma^{6}
\end{array}\right) \\
& X_{c l}=\left(\bar{A} \oplus B \bar{F}_{+}\right)^{*} B V \\
& \left(26 \oplus 35 \gamma^{2} \oplus 47 \gamma^{3} \oplus 53 \gamma^{4} \oplus 59 \gamma^{5}\right. \\
& \text { (cont'd.) } \oplus+\infty \gamma^{6} \\
& 29 \oplus 35 \gamma^{2} \oplus 47 \gamma^{3} \oplus 53 \gamma^{4} \oplus 59 \gamma^{5} \\
& =\quad \begin{array}{c}
\left(\text { cont' }^{\prime} \text { d. }\right) \oplus+\infty \gamma^{6} \\
36 \oplus 42 \gamma \oplus 48 \gamma^{2} \oplus 54 \gamma^{3} \oplus 60 \gamma^{4} \oplus 66 \gamma^{5} \\
\left(\text { cont }^{\prime} \mathrm{d} .\right) \oplus+\infty \gamma^{6}
\end{array} \\
& 29 \gamma \oplus 35 \gamma^{3} \oplus 47 \gamma^{4} \oplus 53 \gamma^{5} \oplus 59 \gamma^{6} \\
& \text { (cont'd.) } \oplus+\infty \gamma^{7} \\
& \left.\begin{array}{c}
26 \gamma \oplus 35 \gamma^{3} \oplus 47 \gamma^{4} \oplus 53 \gamma^{5} \oplus 59 \gamma^{6} \\
\left(\text { cont }^{\prime} \text { d. }\right) \oplus+\infty \gamma^{7}
\end{array}\right) \\
& Y_{c l}=C X_{c l} \\
& =37 \oplus 43 \gamma \oplus 49 \gamma^{2} \oplus 55 \gamma^{3} \oplus 61 \gamma^{4} \oplus 67 \gamma^{5} \oplus+\infty \gamma^{6}
\end{aligned}
$$

Clearly, the output trajectories are equal $Y_{c l}=Y_{o l}$ and $U_{c l} \succeq U_{o l}, X_{c l} \succeq X_{o l}$, i.e., controller $\bar{F}_{+}$is neutral in regards to the output, but delay as much as possible the tokens input.

In a second step, the system is assumed to be disturbed, with $Q=\left(\begin{array}{lll}\varepsilon & 85 \gamma^{3} & \varepsilon\end{array}\right)^{t}$. Entry $Q_{2}=85 \gamma^{3}$ means that the fourth firing occurs at time 85 . This may represents a machine breakdown occurring after the third part be processed and this breakdown lasts until time 85 . The system trajectories without controller $(U=V)$, denoted $U_{o l q}, X_{o l q}$ and $Y_{o l q}$, become

$$
\begin{aligned}
& U_{o l q}=\underline{V}_{*} \\
& X_{\text {olq }}=\bar{A}^{*} B V \oplus \bar{A}^{*} S Q \\
& 26 \oplus 30 \gamma^{2} \oplus 34 \gamma^{4} \oplus+\infty \gamma^{6} \\
& 29 \oplus 35 \gamma^{2} \oplus 85 \gamma^{3} \oplus 91 \gamma^{5} \oplus+\infty \gamma^{6} \\
& =\quad \begin{array}{c}
36 \oplus 42 \gamma \oplus 48 \gamma^{2} \oplus 92 \gamma^{3} \oplus 98 \gamma^{4} \oplus 104 \gamma^{5} \\
\left(\text { cont' }^{\prime} \mathrm{d} \text {. }\right) \oplus+\infty \gamma^{6}
\end{array} \\
& \left.\begin{array}{c}
29 \gamma \oplus 35 \gamma^{3} \oplus 85 \gamma^{4} \oplus 91 \gamma^{6} \oplus+\infty \gamma^{7} \\
26 \gamma \oplus 30 \gamma^{3} \oplus 34 \gamma^{5} \oplus \infty \gamma^{7}
\end{array}\right) \\
& \text { and } \begin{aligned}
Y_{\text {olq }}= & C X_{\text {olq }} \quad 26 \gamma \oplus 30 \gamma^{3} \oplus 34 \gamma^{5} \oplus \infty \gamma^{7} \\
= & 37 \oplus 43 \gamma \oplus 49 \gamma^{2} \oplus 93 \gamma^{3} \oplus 99 \gamma^{4} \oplus 105 \gamma^{5} \\
& \oplus+\infty \gamma^{6}
\end{aligned}
\end{aligned}
$$

Obviously, this machine breakdown delays the firing of transitions $x_{2}$ and $x_{3}$, indeed $X_{o l q} \succeq X_{o l}$ and $Y_{o l q} \succeq Y_{o l}$. With controller $\bar{F}_{+}$, these trajectories, denoted $U_{c l q}, X_{c l q}$ and $Y_{c l q}$ become

$$
\begin{aligned}
& U_{c l q} \quad=\bar{F}_{+} X_{c l q} \oplus V \\
& =\left(\begin{array}{c}
20 \oplus 29 \gamma^{2} \oplus 41 \gamma^{3} \oplus 47 \gamma^{4} \oplus 85 \gamma^{5} \oplus+\infty \gamma^{6} \\
20 \oplus 38 \gamma^{3} \oplus 44 \gamma^{4} \oplus 50 \gamma^{5} \oplus+\infty \gamma^{6}
\end{array}\right) \text {, } \\
& \begin{aligned}
& X_{c l q}=(\bar{A} \oplus B \bar{F}+)^{*} B V \oplus(\bar{A} \oplus B \bar{F}+)^{*} S Q \\
& 26 \oplus 35 \gamma^{2} \oplus 47 \gamma^{3} \oplus 53 \gamma^{4} \oplus 91 \gamma^{5}
\end{aligned} \\
& \text { (cont'd.) } \oplus+\infty \gamma^{6} \\
& 29 \oplus 35 \gamma^{2} \oplus 85 \gamma^{3} \oplus 91 \gamma^{5} \oplus+\infty \gamma^{6} \\
& =\mid \begin{array}{c}
36 \oplus 42 \gamma \oplus 48 \gamma^{2} \oplus 92 \gamma^{3} \oplus 98 \gamma^{4} \oplus 104 \gamma^{5} \\
\left(\text { cont }^{\prime} \text { d. }\right) \oplus+\infty \gamma^{6}
\end{array}, \\
& 29 \gamma \oplus 35 \gamma^{3} \oplus 85 \gamma^{4} \oplus 91 \gamma^{6} \oplus+\infty \gamma^{6} \\
& 26 \gamma \oplus 35 \gamma^{3} \oplus 47 \gamma^{4} \oplus 53 \gamma^{5} \oplus 91 \gamma^{6} \\
& \text { and } Y_{c l q}=C X_{c l q} \\
& \left(\text { cont' }^{\prime} \text { d. }\right) \oplus+\infty \gamma^{7} \\
& =37 \oplus 43 \gamma \oplus 49 \gamma^{2} \oplus 93 \gamma^{3} \oplus 99 \gamma^{4} \oplus 105 \gamma^{5} \\
& \oplus+\infty \gamma^{6} \text {. }
\end{aligned}
$$

The output $Y_{c l q}=Y_{\text {olq }}$, i.e., the controller $\bar{F}_{+}$does not disturb the system, nevertheless $X_{c l q} \succeq X_{o l q}$ and $U_{c l q} \succeq$ $U_{\text {olq }}$ this means that the tokens input is delayed. Furthermore this is done in an optimal manner, then the input of useless tokens is avoid.

\section{CONCLUSiON}

This paper reports upon recent investigations on the disturbance decoupling problem for max-plus linear systems as well as the corresponding solvability conditions and controller synthesis procedures. Future research directions along the geometric control of max-plus linear systems are block decoupling problem and model matching problem.

\section{REFERENCES}

[1] Baccelli, F., Cohen, G., Olsder, G.J., \& Quadrat, J.-P. (1992) Synchronization and Linearity: An Algebra for Discrete Event Systems. New York: John Wiley and Sons. http://www-rocq.inria. $\mathrm{fr} / \mathrm{metalau} / \mathrm{cohen} / \mathrm{SED} / \mathrm{SED} 1$-book. html

[2] Cohen, G., Gaubert, S., \& Quadrat, J.-P. (1996) Kernels, images, an projections in dioids, Proceedings of WODES'96, Edinburgh.http: / www-rocq.inria.fr/metalau/quadrat/kernel.pdf

[3] Cohen, G., Gaubert, S., \& Quadrat, J.-P. (1997) Linear projectors in the max-plus algebra. The 5th IEEE-Mediterranean Conference, Paphos, Cyprus. http://www-rocq.inria.fr/metalau/quadrat projector.pdf

[4] Cohen, G., Gaubert, S., \& Quadrat, J.-P. (1999) Max-plus algebra and system theory: where we are and where to go now. Annual Reviews in Control, 23, 207-219.

[5] Cohen, G., Gaubert, S., \& Quadrat, J.-P. (2006) Projection and Aggregation in Maxplus Algebra, Current Trends in Nonlinear Systems and Control, in Honor of Peter Kokotovic and Turi Nicosia Boston: Birkhäuser. http://www-rocq.inria.fr/metalau/ quadrat/Rome.pdf

[6] Cottenceau, B., Hardouin, Lhommeau, M. \& Boimond, J.-L. (2000) Data processing tool for calculation in dioid, The 5th Workshop on Discrete Event Systems, Ghent, Belgium, http://www.istia. univ-angers.fr/ hardouin/outils.html

[7] Cottenceau, B., Hardouin, L., Boimond, J.-L., \& Ferrier,J.-L. (2001) Model reference control for timed event graphs in dioid. Automatica, 37, $1451-1458$

[8] De Jong, H. (2002) Modeling and simulation of genetic regulatory systems: a literature review. J. Computational Biology, 9, 1, 67-103.

[9] Di Loreto, M., Gaubert, S., Katz, R. \& Loiseau, J-J, (2010) Duality between invariant spaces for max-plus linear discrete event systems, 48, 5606-5628, http://fr.arXiv.org/abs/0901.2915.

[10] Golan, J.S. (1999) Semirings and Their Applications. Boston: Kluwer Academic Publishers.

[11] Hardouin, L., Maia, C.A., Cottenceau, B, \& Lhommeau, M. (2010) Observer Design for (max plus)-Linear Systems, IEEE Transaction on Automatic Control, 55, 2, 538-543.

[12] Katz, R.D. (2007) Max-plus (A.B)-invariant spaces and control of timed discrete-event systems, IEEE Trans. Auto. Control, 52, 2, 229241

[13] Le Boudec, J.-Y. \& Thiran, P. (2002) Network Calculus. New York: Springer-Verlag.

[14] Lhommeau, M.; Hardouin, L., \& Cottenceau, B. (2002) About disturbance decoupling of timed event graphs in dioids. The 6th International Workshop on Discrete Event Systems, pp. 203-208. http:// ww. istia.univ-angers. fr/ $\sim$ hardouin/Wodes 02 .pdf

[15] Lhommeau, M.; Hardouin, L., \& Cottenceau, B. (2002) Disturbance decoupling of timed event graphs by output feedback controller, The 6th Workshop on Discrete Event Systems Zaragoza, Spain, http: / / www. istia.univ-angers. fr/ hardouin/Wodes 02 . pdf

[16] Lhommeau, M.; Hardouin, L., \& Cottenceau, B. (2003) Optimal control for (max,+)-linear systems in the presence of disturbances, Positive Systems: Theory and Applications, POSTA, Springer LNCIS 294,47-54.

[17] Maia, C.A, Andrade, C.R. \& Hardouin, L. (2011) On the control of max-plus linear system subject to state restriction. Automatica, 47-5, 988-992.

[18] Maia, C.A, Hardouin,L., Santos-Mendes, R., \& Cottenceau, B.(2003 Optimal closed-loop control of timed event graphs in dioids. IEEE Transactions on Automatic Control, 49, 12, 2284-2287.

[19] Maia, C.A, Hardouin,L., Santos-Mendes, R., \& Cottenceau, B.(2005) On the Model reference control for max-plus linear systems. The 44th IEEE Conference on Decision and Control, pp. 7799-7803.

[20] Prou, J.-M., \& Wagneur, E. (1999) Controllability in the max-algebra. KYBERNETIKA. 35, 1, 13-24.

[21] Wonham, W.M. (1979) Linear Multivariable Control: A Geometric Approach. New York: Springer-Verlag. 